\title{
SYNTHESIS, CHARACTERIZATION AND COMPUTATION OF POTASSIUM DOPED CALCIUM HYDROXIDE NANOPARTICLES AND NANOTUBES
}

\author{
D. NANDHINI ${ }^{1}$, S. SUBASHCHANDRABOSE ${ }^{1}$, P. RAMESH ${ }^{1}$, \\ D. MOHAN RADHEEP ${ }^{1,2} \&$ K. SAKTHIPANDI ${ }^{3}$ \\ ${ }^{I}$ Centre for Research and Development, PRIST Deemed to be University, Vallam, Thanjavur, Tamil Nadu, India \\ ${ }^{2}$ Department of Physics, PRIST Deemed to be University, Puducherry Campus, Puducherry, India \\ ${ }^{3}$ Department of Physics, Sethu Institute of Technology, Kariapatti, Tamil Nadu, India
}

\begin{abstract}
Potassium doped calcium hydroxide [Ca(OH)2:K] nanoparticles were synthesized by simple precipitation method. The powder $x$-ray diffraction pattern of the sample was studied, to investigate the crystalline nature of the sample. The shape and size of the nanoparticles were measured by scanning electron microscopic (SEM). The functional groups presented in the synthesized particles were recorded and analysed in the spectral region of $4000-400 \mathrm{~cm}^{-1}$ by Fourier-transform infrared spectroscopy. Optical Properties of $[\mathrm{Ca}(\mathrm{OH}) 2: \mathrm{K}]$ were determined by ultraviolet visible spectrometer in the range of $190-800 \mathrm{~nm}$. In addition that to study insight of the $\mathrm{Ca}(\mathrm{OH}) 2$ nano material, a nanotube was constructed and studied a quantum chemical calculations to predict the geometry, Dynamic and band gap properties.

KEYWORDS: Potassium Doped Calcium Hydroxide; Structural Properties \& Optical Properties
\end{abstract}

Received: Dec 06, 2018; Accepted: Dec 26, 2018; Published: Jan 22, 2019; Paper Id.: IJMPERDFEB201943

\section{INTRODUCTION}

In recent years, a widespread research has been made on calcium hydroxide $(\mathrm{Ca}(\mathrm{OH}) 2)$ nanoparticle $(\mathrm{NP})$, since it has a vital applications various fields. $\mathrm{Ca}(\mathrm{OH}) 2$ NPs has numerous applications such as paper de-acidification, wall painting, consolidation of artwork, natural stones, wood, cultural heritage conservation [1-5], $\mathrm{CO}_{2}$ adsorption of greenhouse gas and endodontic [6-8]. Since it has potential applications, we are interested in investigating the optical behaviour of $\mathrm{Ca}(\mathrm{OH}) 2$ when doped with potassium $(\mathrm{K})$. In the present study, we synthesize potassium-doped Calcium hydroxide $(\mathrm{Ca}(\mathrm{OH}) 2: \mathrm{K})$ nanoparticle and characterize the structure, optical band gap and surface morphology (SEM). In addition to that using quantum chemical, DFT and Td-DFT method of calculations a $\mathrm{Ca}(\mathrm{OH}) 2$ nanotube was studied, it provides as detailed information about geometry, band gap and a vibration picture of the $\mathrm{Ca}(\mathrm{OH}) 2$ NPs. Besides that to investigate the functional groups and optical gap the FT-IR spectrum and optical absorbance UV-Visible spectrum was recorded.

\section{EXPERIMENTAL DETAILS}

Materials and Methods

Chemicals and reagents were used in this study were analytical grade and used without further Purification. Calcium chloride $\left(\mathrm{CaCl}_{2}\right.$, Merck), Sodium hydroxide $(\mathrm{Na}(\mathrm{OH})$, Merck), Potassium Chloride ( $\mathrm{KCl}$, Merck) were used as starting materials. 
Synthesis of Potassium Doped Calcium Hydroxide

The $\mathrm{Ca}(\mathrm{OH})_{2}$ doped with $\mathrm{K}$ doped nanoparticles were synthesized by the following reaction:

$\mathrm{CaCl}_{2}+2 \mathrm{NaOH}+\mathrm{KCl} \rightarrow \mathrm{Ca}(\mathrm{OH})_{2} \mathrm{~K}+2 \mathrm{NaCl}$

$\mathrm{CaCl}_{2}, 3.3 \mathrm{~g}$ and $\mathrm{NaOH}, 2.4 \mathrm{~g}$ was dissolved in $100 \mathrm{ml}$ of double distilled water and stirred for $15 \mathrm{~min}$. At $70^{\circ} \mathrm{C}$ to obtain a clear solution, the cyclohexane was added into the prepared clear solution and maintained in a beaker. In the meantime, $0.7 \mathrm{~g}$ of potassium chloride was dissolved in $20 \mathrm{ml}$ of distilled water and added into the beaker by drop wise under vigorous stirring. The mixture was maintained at the standard temperature. The mixture was centrifuged and washed with water, then dried at $60^{\circ} \mathrm{C}$ using the micro oven. Finally, the white colour powder was obtained.

\section{Characterization and Instrumentation}

The potassium doped calcium hydroxide was studied using powder x-ray diffraction method. $\mathrm{Ca}(\mathrm{OH}) 2: \mathrm{K}$ was dissolved in de-ionized water, and its optical behaviour was examined bya UV spectrophotometer (systronics) (Perkin-Elmer lambda 25 UV spectrometer). The Fourier-transform infrared spectrum (FTIR) of the powdered solution was recorded in $\mathrm{KBr}$ medium in the range of 400-4000 $\mathrm{cm}^{-1}$ using Perkin Elmer RX1 infrared scanner. The morphology, size distribution, and shape of the prepared nanoparticles were observed by scanning Electron Microscopy (SEM).

\section{Computational Details}

Complementary density functional theory (DFT) and Time-dependent DFT calculations were performed using Gaussian 03 program [9, 10]. The B3LYP/LANL2DZ basis was used to calculate the molecular geometry, vibrational frequencies, and electronic excitations. Initially, a minimum size of $\mathrm{Ca}(\mathrm{OH}) 2$ nanotube was constructed in the range about $0.344 \mathrm{~nm}$ of breadth and $0.666 \mathrm{~nm}$ of length. The nanotube consists of $6: 6$ ratio oxygen and calcium hydroxide atoms in Hexagonal shape [11]. The optimized structure of $\mathrm{NT}$ of $\mathrm{Ca}(\mathrm{OH}) 2$ is shown in Figure 1.

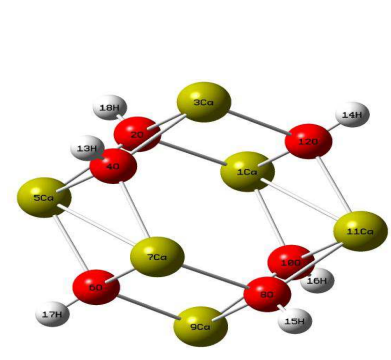

(a)

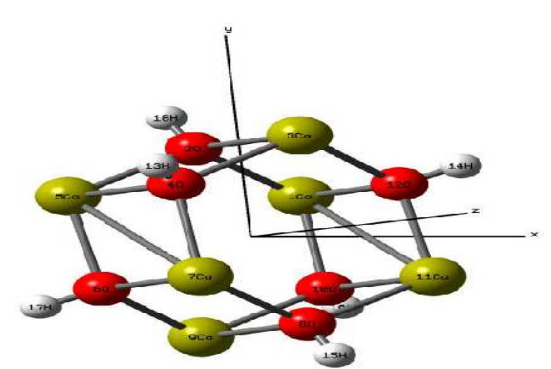

(b)

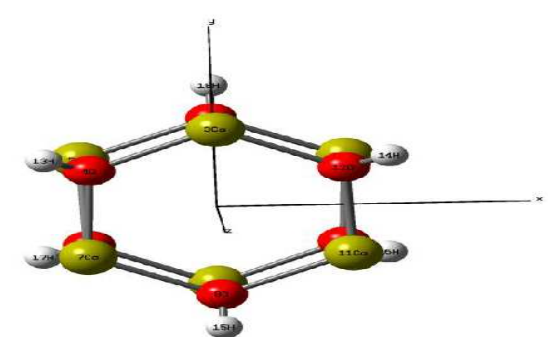

(c)

Figure 1: The Optimized $\mathrm{Ca}(\mathrm{OH}) 2$ nanotube(3,3) by B3LYP/LANLD2Z Basis Seta) The Hexagonal Shape of NPs, b) Vertical View of the NPs

c) NPs is Horizontal View 


\section{RESULTS AND DISCUSSION}

The geometry calculation of $\mathrm{Ca}(\mathrm{OH})_{2}$ nanotube performed with the hexagonal packing system. Atomic bonding appears as $\mathrm{Ca}-\mathrm{O}, \mathrm{Ca}-\mathrm{Ca}$, and $\mathrm{O}-\mathrm{H}$, its bond lengths calculated in angstrom $(\AA)$. The bond length of $\mathrm{Ca}-\mathrm{O}_{2}$ is about $2.425 \AA$ whereas $\mathrm{Ca} 3-\mathrm{O}_{4}$ and $\mathrm{Ca} 3-\mathrm{O}_{12}$ have slightly lesser $(2.368$ and $2.369 \AA)$ than that, it is due to the positioning of atoms, the $\mathrm{Ca} 1-\mathrm{O}_{2}$ is a bonding between the hexagon and whereas the counterpart is within the hexogen. On the whole, Ca-O has a lesser bond length along the line joining position in the nanotube. The $\mathrm{Ca}-\mathrm{Ca}$ bonding was calculated higher than $\mathrm{Ca}-\mathrm{O}$ bond lengths about $4.229 \AA$ caused its interaction due to inter-bonding between ortho and para-positioned atoms. The optimized bond parameters are presented in Table 1.

Table 1: The Optimized Bond Parameters Of $\mathrm{Ca}(\mathrm{OH}) 2$ Nanotube, Using B3LYP/LANL2DZ Basis Set

\begin{tabular}{|l|c|c|c|c|c|}
\hline \multicolumn{2}{|c|}{ Parameters Bond Length $(\AA)$} & \multicolumn{2}{c|}{ Parameters Bond Length $(\AA)$} & \multicolumn{2}{c|}{ Parameters Bond Length $(\AA)$} \\
\hline Ca1-O2 & 2.425 & Ca1-Ca3 & 3.697 & O2-H18 & 0.985 \\
\hline Ca1-O12 & 2.391 & Ca1-Ca5 & 4.229 & O4-H13 & 0.987 \\
\hline O2-Ca3 & 2.451 & Ca1-Ca9 & 4.097 & O6-H17 & 0.987 \\
\hline O2-Ca5 & 2.425 & Ca1-Ca11 & 3.634 & O8-H15 & 0.985 \\
\hline Ca 3-O4 & 2.368 & Ca3-Ca5 & 3.698 & O10-H16 & 0.987 \\
\hline Ca3-O12 & 2.369 & Ca3-Ca7 & 4.093 & O12-H14 & 0.987 \\
\hline O4- Ca5 & 2.391 & Ca3-Ca11 & 4.095 & & \\
\hline Ca5-O6 & 2.390 & Ca5-Ca7 & 3.635 & & \\
\hline O6-Ca7 & 2.391 & Ca5-Ca9 & 4.093 & & \\
\hline Ca7-O8 & 2.425 & Ca7-Ca9 & 3.697 & & \\
\hline O8-Ca9 & 2.451 & Ca7-Ca11 & 4.229 & & \\
\hline Ca9-O10 & 2.368 & Ca9-Ca11 & 3.697 & & \\
\hline O10-Ca11 & 2.391 & & & & \\
\hline Ca11-O12 & 2.390 & & & & \\
\hline
\end{tabular}

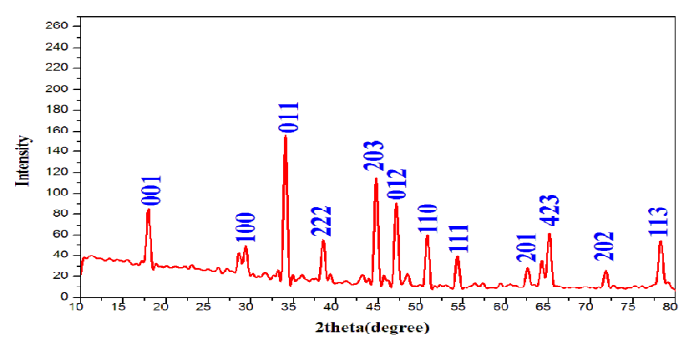

(a): The Powder X-Ray Pattern of $\mathrm{Ca}(\mathrm{OH}) 2$ : K

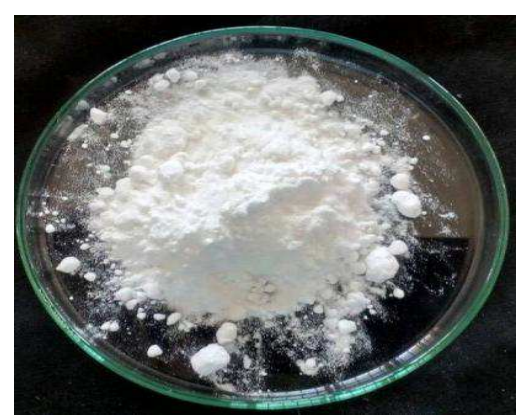

(b) The White Colored Powder Sample of $\mathrm{Ca}(\mathrm{OH}) 2$ nanoparticles Figure 2: The Powder X-ray Pattern and Synthesized Sample of $\mathrm{Ca}(\mathrm{OH}) 2: \mathrm{K}$ 
The XRD pattern and the synthesized sample have shown in Figure 2. The pattern indicates that the crystalline nature and miller indices of $\mathrm{Ca}(\mathrm{OH}) 2$, appeared as (001), (100), (110) and (111), it confirms the indexed hexagonal structure $\mathrm{Ca}(\mathrm{OH}) 2[12,13]$, the $2 \theta$ degrees appears at 18.13, 29.49, 50.82, and 54.33. The Potassium doping in $\mathrm{Ca}(\mathrm{OH}) 2$ was confirmed, due to the presence of miller indices (011), (222) and (202) at a $2 \theta$ angle of 34.10, 38.52 and 71.94 respectively. The SEM image of pure potassium doped calcium hydroxide nanoparticles shows hierarchical nanostructure of flower-like morphology with a mean diameter of $2 \mu \mathrm{m}$, which consist flower and rod-shaped nanostructures as shown in Figure 3.
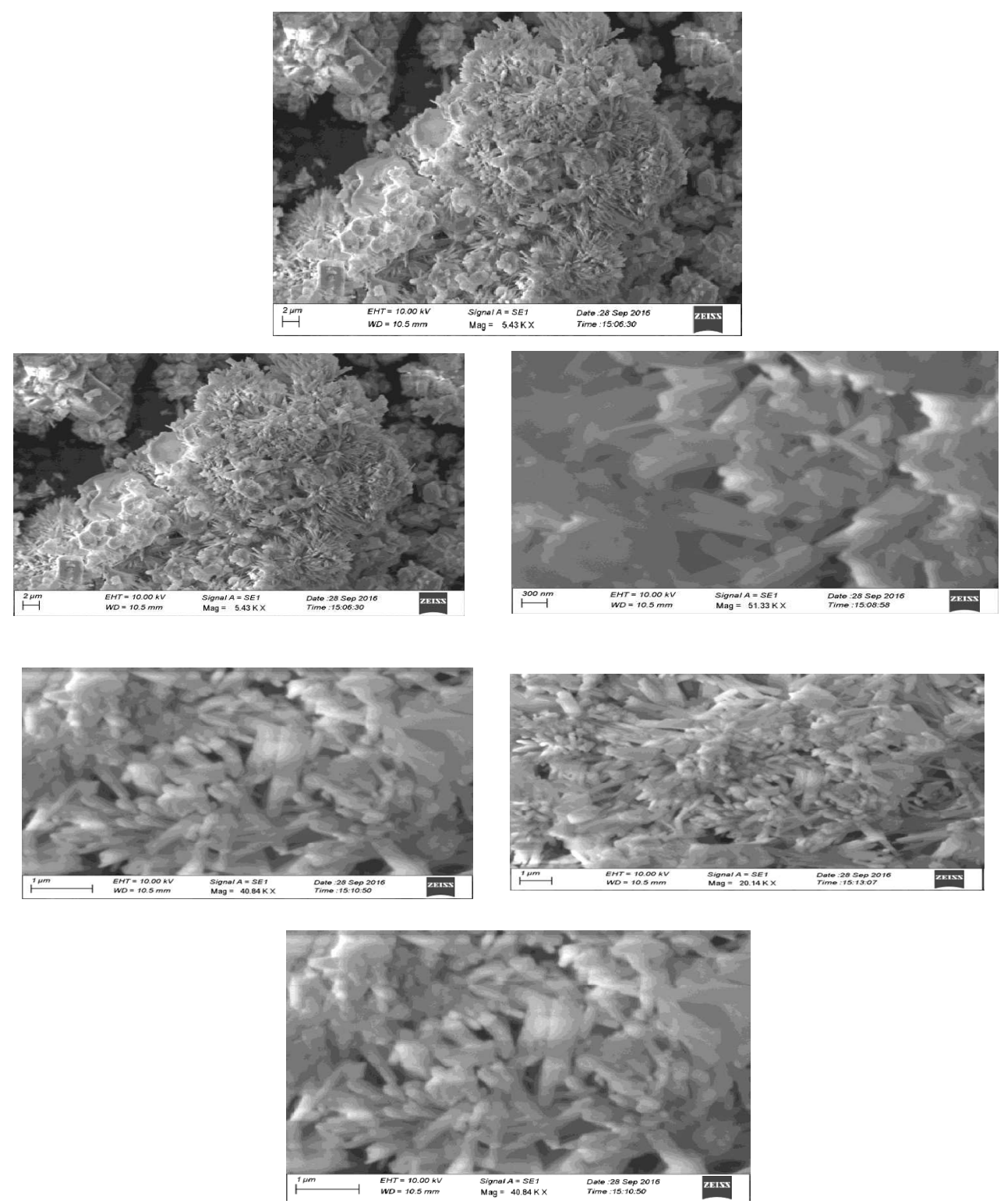

Figure 3: SEM Analysis of Potassium Doped $\mathrm{Ca}(\mathrm{OH}) 2$ nanoparticles

It is exactly matched with traditional $\mathrm{Ca}(\mathrm{OH}) 2$ nanoparticles structure [12 14]. The $\mathrm{Ca}(\mathrm{OH}) 2 \mathrm{NPs}$ has a hexagonal shape with several nanometers in thickness $(40-45 \mathrm{~nm})$ and breath $(600-650 \mathrm{~nm})$ [14]. In the present investigation, we obtained an exact sign of $\mathrm{Ca}(\mathrm{OH}) 2: \mathrm{K}$ NPs in different magnification. Further FT-IR spectrum was recorded (as shown in Figure 4) 


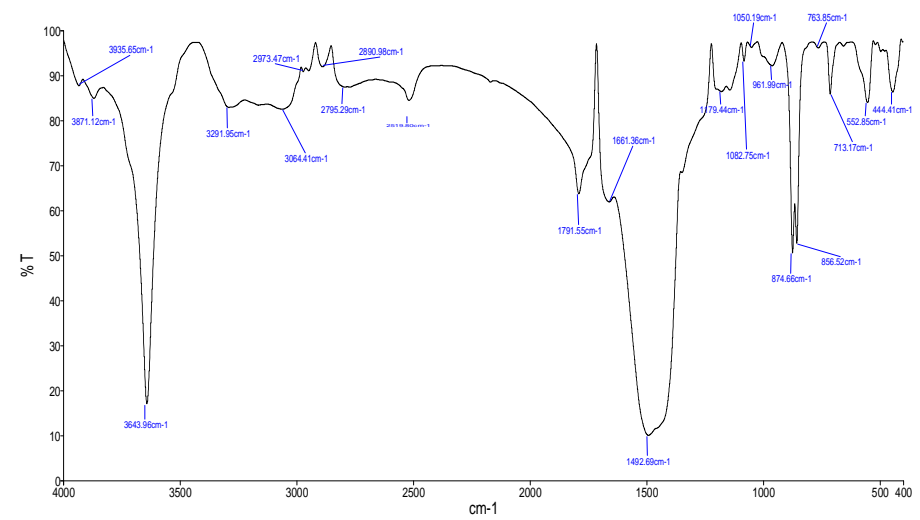

Figure 4: The Experimental FT-IR Spectrum of $\mathrm{Ca}(\mathrm{OH}) 2: \mathrm{K}$

To find the presence of functional groups in the range of $4000-400 \mathrm{~cm}^{-1}$, the vibrational picture of the $\mathrm{Ca}(\mathrm{OH}) 2: \mathrm{K}$ NPs sample shows a peak at $3643.96 \mathrm{~cm}^{-1}$ belongs to O-H stretching band as a sharp peak [12]. A counterpart computed wave numbers appear at 3498 and $3456 \mathrm{~cm}^{-1}$, it shows a deviation (145 and $\left.187 \mathrm{~cm}-1\right)$ due to the chemical environment. The peak at $1661 \mathrm{~cm}^{-1}$ indicates the bending of the O-H band, this peak shows a small difference from the literature [12], it is due to the Potassium element presented in the material. A peak appeared at $875 \mathrm{~cm}^{-1}$ is belongs to $\mathrm{CaO}_{3}$, as a strong band with a double peak of $857 \mathrm{~cm}^{-1}$. Also, a medium intensity peak of $\mathrm{CaO}$ stretching has obtained at $444 \mathrm{~cm}^{-1}$, whereas the calculated wave number of the same mode has obtained at $359 \mathrm{~cm}^{-1}$. The wagging in plan bending vibration of $\mathrm{Ca}(\mathrm{OH}) 2$ has appeared at $553 \mathrm{~cm}^{-1}$, its corresponding computed values is about $564 \mathrm{~cm}^{-1}$, it shows good agreement among both the methods. The recorded and computed wave numbers were presented in Table 2. The computed wave numbers were scaled down by 0.961 to bring the computed results near with observed Values as suggested by CCCBDB [15].

\section{Table 2: The FT-IR and B3LYP/LANL2DZ Basis Computed Wave Number of $\mathrm{Ca}(\mathrm{OH})_{2}: \mathrm{K}$ and Computed $\mathrm{Ca}(\mathrm{OH})_{2}$ NTS (Scale Factor: 0.961)}

\begin{tabular}{|c|c|c|c|}
\hline Un Scaled $\left(\mathbf{c m}^{-1}\right)$ & Scaled $\left(\mathbf{c m}^{-\mathbf{1}}\right)$ & FT-IR $\left(\mathbf{c m}^{\mathbf{- 1}}\right)$ & Assignments \\
\hline 373.58 & 359 & 444 & $\mathrm{vCaO}$ \\
\hline 559 & 537 & & $\omega \mathrm{CaOH}$ \\
\hline 586.61 & 564 & 553 & $\omega \mathrm{CaOH}$ \\
\hline 714.91 & 687 & 713.17 & $\mathrm{tCaOH}$ \\
\hline 3596.39 & 3456 & & $\nu \mathrm{OH}$ \\
\hline 3639.64 & 3498 & & $\mathrm{vOH}$ \\
\hline 3640.33 & 3498 & 3643.96 & $\mathrm{vOH}$ \\
\hline
\end{tabular}

The UV absorption spectrum was recorded in the range of 190-1100 nm and its shown in Figure 5, there is a peak appeared at $235 \mathrm{~nm}$ due to the absorption of $\mathrm{Ca}(\mathrm{OH})_{2}: \mathrm{K}$ nanoparticles. The peak has emerged in the conjugative range of $\pi-\pi^{*}$ transition and it reflects the optical behaviour of the material. On the other hand, the predicted Td-B3LYP calculation the band gap of $\mathrm{Ca}(\mathrm{OH}) 2 \mathrm{NT}$ is calculated about $-0.4696 \mathrm{eV}$. It is the difference between highest occupied molecular orbital (HOMO) and lowest unoccupied molecular orbital (LUMO), HOMO has appeared over the ortho-meta position of the hexagonal and LUMO located over the Para position of the hexagon. The closer band gap among HOMO and LUMO indicates the ability to react with a substituent. This kind of materials is very suitable for Endodontic [8]. The frontier molecular structure of HOMO-LUMO has shown in Figure 6 

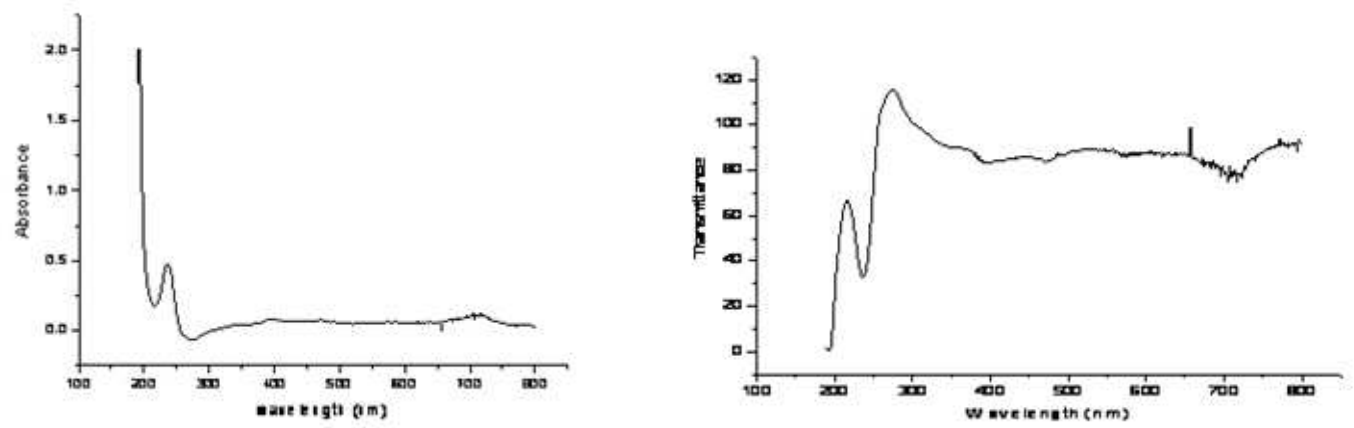

Figure 5: UV Absorbance and Transmittance of Potassium Doped $\mathrm{Ca}(\mathrm{OH}) 2$ nanoparticles

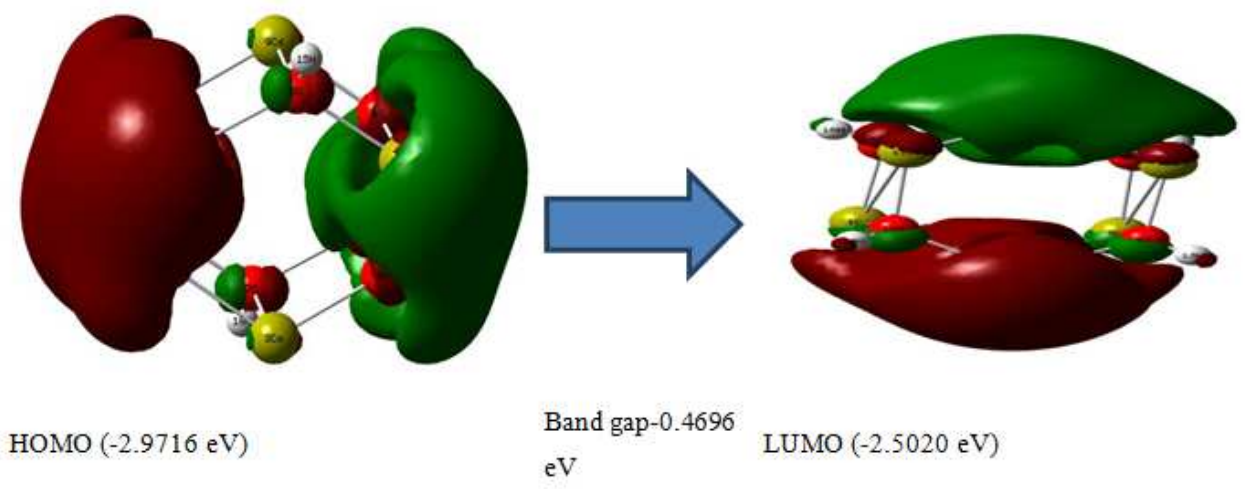

Figure 6: The Frontier Molecular Orbital's of $\mathrm{Ca}(\mathrm{OH}) 2$ NT Using Td-DFT Calculation

\section{CONCLUSIONS}

The potassium doped Calcium hydroxide nanoparticles were prepared using the wet chemical method. The atomic plane was found using powder X-ray diffraction method, miller indices of $\mathrm{Ca}(\mathrm{OH}) 2$ : K were (001), (100), (110), (111), (011), (222) and (202). The SEM image analysis confirmed the flower and rod shape morphology of the potassium doped calcium hydroxide nanoparticles with $2 \mu \mathrm{m}$ diameter. In FTIR, the typical hydroxyl group and Ca-O stretching, and $\mathrm{Ca}-\mathrm{O}-\mathrm{Ca}$ bending vibrations were found at a characteristic region. The UV-Visible spectrum dealt the optical behaviour of NPs and NTs, absorbed optical gap is in the conjugative region $(235 \mathrm{~nm})$, where exist $\pi-\pi^{*}$ transition between $\mathrm{p}$ and $\mathrm{d}$ orbital electrons. The calculated band gap of NT is about $-0.4696 \mathrm{eV}$, shows a good sign of reactivity of the material.

\section{REFERENCES}

1. R. Giorgi, D.Chelazzi, P.Baglioni, Langmuir, 21 (2005)10743-10748.

2. C. Rodriguez-Navarro, A.Suzuki, E.Ruiz-Agudo, Langmuir, 29 (2013) 11457-11470.

3. V. Daniele and G.Taglieri, J.CulturalHerit.13 (2012) 40-46.

4. E. Carretti, D.Chelazzi, G.Rocchigiani, P.Baglioni, G.Poggi, L.Dei, Langmuir 29 (2013)988- 989.

5. R. Giorgi, M.Baglioni, D.Berti, P.Baglioni, Acc.Chem.Res.43 (2010) 695-704.

6. N. N. Hlaing, R. Othman, H. Hinode, W. Kurniawan, A. A. Thant, A. R. Mohamed, C. Salim andS. Sreekantan, Procedia Environ. Sci., 20 (2014) 71-78.

7. V. Materic and S. I. Smedley, Ind. Eng. Chem. Res., 50 (2011) 5927-5932. 
8. N. N. Hlaing, S. Sreekantan, R. Othman, H. Hinode, W. Kurniawan, A. A. Thant, A. R.Mohamed and C. Salim, Mater. Lett., 133 (2014) 204-207.

9. M.J. Frisch, Gaussian 03, Revision C.02, Gaussian Inc., Wallingford, CT, 2004.

10. A. D. Becke, Phys. Rev. A.,38 (1988) 3098-3100.

11. J. T. Frey and D. J. Doren, University of Delaware, Newark DE, 2011.

12. M.Darroudi, M. Bagherpour, H.A. Hosseini, M.Ebrahimi, Ceramics Int. 42 (2016) 3816-3819.

13. Krishnamoorthi, S., Prabhahar, M., Kumar, M. S., \& Sendilvelan, S. (2018). Yield Characteristic of Biodiesel Derived From Used Vegetable Oil Methyl Ester (UVOME) Blended With Diesel, in the Presence of Sodium Hydroxide (NAOH) and Potassium Hydroxide (KOH) Catalyst, As Alternative Fuel for Diesel Engines. 8(1), 9-16.

14. J.A. Madrid, M. Lanzón, Appl. Surface Science 424 (2017) 20-27.

15. F.D. M. Daud, K.Vignesh, S. Sreekantan, A.R. Mohamed, M. Kang and B.S. Kwake, RSC Adv., (2016) 36031-36038.

16. Yadav, D., Singh, S., \& Singh, S. Effect of Foliar Application of Potassium Compounds on Yield And Quality of Ber (Zizyphus Mauritiana Lam.) Cv. Banarasi Karaka.

17. Russell D. Johnson III (Ed.), NIST Computational Chemistry Comparison and Benchmark Database, NIST Standard Reference Database Number 101, Release, 17b, September 2015. 
